\title{
Constructing Kinetically Controlled Denaturation Isotherms of Folded Proteins Using Denaturant-Pulse Chaperonin Binding
}

\author{
Pierce T. O'Neil ${ }^{1}$, Alexandra J. Machen ${ }^{1}$, Jackie A. Thompson ${ }^{1}$, Wei Wang ${ }^{2}$, Quyen $Q$. \\ Hoang $^{2}$, Michael R. Baldwin ${ }^{3}$, Karen R. Khar ${ }^{4}$, John Karanicolas ${ }^{4}$, and Mark T. Fisher ${ }^{1}$ \\ ${ }^{1}$ Department of Biochemistry and Molecular Biology, University of Kansas Medical Center, \\ Kansas City, KS, USA \\ 2Department of Biochemistry and Molecular Biology, Indiana University School of Medicine, \\ Indianapolis, IN 46202 \\ ${ }^{3}$ Michael R. Baldwin, Department of Molecular Microbiology and Immunology, University of \\ Missouri, Columbia, MO, USA \\ ${ }^{4}$ Molecular Therapeutics Program, Fox Chase Cancer Center, Philadelphia, Pennsylvania 19111, \\ United States
}

\section{Abstract}

Methods to assess the kinetic stability of proteins, particularly those that are aggregation prone, are very useful in establishing ligand induced stabilizing effects. Because aggregation prone proteins are by nature difficult to work with, most solution based methods are compromised by this inherent instability. Here, we describe a label-free method that examine the denaturation of immobilized proteins where the dynamic unfolded protein populations are captured and detected by chaperonin binding.

\section{Keywords}

Denaturant Pulse; Biolayer Interferometry; Protein Folding; GroEL; Denaturation; Aggregation; Chaperonin

\section{Introduction}

The use of protein denaturation procedures coupled with an appropriate detection output (e.g. spectroscopic; calorimetric thermophoresis) allows researchers to assess the impact of ligand binding to proteins both stabilizing and destabilizing and to compare the stability of mutant proteins with their wild type counterparts. These methods using temperature or chaotrope perturbation work well for well-behaved proteins where their denaturation profiles are not complicated by extensive competing aggregation side reactions. Unfortunately, a large number of proteins for which protein denaturation profiles are used to assess protein stability undergo extensive aggregation under physiologically relevant conditions. This is

Send Correspondence to: Mark T. Fisher, Department of Biochemistry and Molecular Biology, University of Kansas Medical Center, 3901 Rainbow Boulevard, Kansas City, KS 66160-7421. Tel: 913-588-6940., mfisher1@kumc.edu. 
particularly problematic for assessing the validity of potentially therapeutic stabilizing ligands of less stable disease proteins that undergo pathological unfolding or aggregation. These aggregation side reactions interfere with the reproducibility of isotherms. If one simply relies on scoring those candidate ligands that prevent the off-pathway aggregation, there is the potential for identifying false positive candidate ligands when the small molecule simply inhibits the aggregation reaction rather than truly stabilizing the "native"-like fold.

In this methods monograph, expanded and detailed protocols are provided to easily construct denaturant isotherms using a label-free platform. The methods involve immobilizing target proteins on a biosensor surface which has a two-fold purpose. First, immobilization of the less stable proteins prevents aggregation side reactions which are a confounding factor for solution-based assays. Second, the protein is oriented in a manner to allow for the adequate readout detection of exposed hydrophobic surfaces formed during a timed denaturant pulse using the large nonspecific chaperonin GroEL to amplify the signal. In this instance, the readout is the detection of chaperonin binding, which serves as a "promiscuous antibody" to partially folded proteins. Due its large size ( $802 \mathrm{kDa})$, GroEL binding is easily discerned using label-free approaches. Reproducibility in the binding readout is obtained using automated, precisely timed GroEL interaction and wash phases. In this work, new examples of utility and process development are presented, including pre-runs of an alpha-synuclein construct, demonstrating cooperative unfolding, supporting the notion that alpha-synuclein contains a folded region (alpha helical interacting regions). In addition, vastly improved reproducible isotherms are generated with increasing the purity of the protein of interest, strengthening the assessment that these methods can distinguish between destabilized and ligand-stabilized forms during small molecule development for protein stabilization.

The label-free technique utilized in this monograph is Biolayer Interferometry (BLI), which is similar to Surface Plasmon Resonance (SPR). The biosensor contains both a reference layer and the biocompatible surface layer. White light is directed down the biosensor and is reflected back up by both layers. The difference in the reflection from both layers creates an interference pattern. As the mass on the biocompatible surface layer changes, the interference pattern maxima change. Monitoring the nanometer shifts in maxima is recorded as the response versus time by the BLI instrument.

\section{Materials}

2.1 Specific Materials Required:

1. Octet RED96 - FortéBio.

2. Software: Data Acquisition 8.2 \& Data Analysis 8.2 - FortéBio. The exact programming may change with later versions of the programs. The screenshots and descriptions given in this monograph are specific for version 8.2.

3. Ni-NTA Biosensors - FortéBio.

4. 96 Well Non-binding Microplates, Non-Sterile - Greiner Bio-One \#655900. Note that plates MUST be black and opaque so as to not 
reflect projected light or transmit outside light. Additionally, this nonbinding property is required so that the Protein of Interest (POI) does not adhere to the well itself, effectively changing the functional concentration.

2.2 p53 Buffer: 50mM TRIS pH 7.5, 300mM NaCl, 1mM DTT. p53 Buffer is used as an example of a POI buffer. It is generally prepared as a 10X stock solution without DTT and upon dilution with ultra-pure $(>18.2 \mathrm{M} \Omega)$ water to working concentration DTT is added. This stock solution is especially useful when preparing solutions containing denaturant.

2.3 GroEL Buffer: 50mM TRIS pH 7.5, 50mM KCl, $10 \mathrm{mM} \mathrm{MgCl}_{2}, 0.5 \mathrm{mM}$ EDTA. GroEL Buffer is used during the denaturant pulse assay as a readout of a protein's hydrophobic exposed nature. This buffer is generally prepared as a 10X stock solution and then diluted with ultra-pure water.

2.4 8M Urea Stock: $8 \mathrm{M}$ urea, $1 \mathrm{x}$ POI buffer. The initial denaturant is urea as it is both chaotropic and non-ionic. The urea stock solution can be made no longer than 12 hours before its use as it degrades in solution into ammonia and isocyanic acid. Storage on ice is recommended to limit degradation, but stock solutions will reversibly precipitate under $20^{\circ} \mathrm{C}$.

2.5 The $10 \mathrm{mM}$ Glycine, $\mathrm{pH} 1.7$ biosensor regeneration solution is generally prepared as a dilution of a 10X stock solution. The stock solution is prepared as $100 \mathrm{mM}$ Glycine in ultra-pure water with its $\mathrm{pH}$ adjusted to 1.7. Dilution into ultra-pure water does not significantly impact $\mathrm{pH}$.

2.6 The $10 \mathrm{mM} \mathrm{NiCl}_{2}$ biosensor regeneration solution is generally prepared as a dilution of a 10X stock solution in ultra-pure water.

\section{Methods}

\subsection{Programming the Octet}

1. Check the power state of the Octet RED96 and the light source. If off, turn both on now as the light source requires at least 1 hour to warm up, preferably 4 hours. By the time the system has been programmed, the biosensors readied, and the assay plate pipetted, at least 1 hour should have passed.

2. Open the Data Acquisition software provided with the Octet. In the Experiment Wizard window, select New Kinetics Experiment and click the arrow. In the first tab of the new window, program the "Plate Definition" for the denaturant pulse automated assay into the computer according to Figure 1. This is accomplished by right clicking on the column number and selecting the proper column type then typing the in the sample ID in the right pane. This example protocol is for His-tagged p53 and biosensor regeneration. Adjust the buffer composition in Columns $1-4$ for your POI. However, the composition of Columns $6-8$ should not be adjusted as this is the best buffer for GroEL binding. 
3. In the next tab, program the "Assay Definition" using the Plate Definition created in step 3.1.2. Add the steps shown in the top right pane using the proper time in seconds and step type as shown in Figure 2. To program the assay steps, click on the Step and then double click on the appropriate column on the plate. Once all 15 steps shown have been programed, select all of them and click replicate button. In the new window selected "Append to current assay" radio option and deselect, if necessary, the checkbox for "Offset steps". Repeat these steps so that the assay is repeated three times in a row which will give an $n=3$ for statistics and error bars. The example is for p53 denaturant pulse but is appropriate for most proteins. The Denaturant Pulse step length can be adjusted per Note 1. The short baseline step (Step 5 in Figure 1) is a brief wash time to remove the denaturant but may permit partial refolding and can be adjusted (Note 2).

4. In the third tab, ensure the correct Sensor Assignment settings are selected (Figure 3). In the top left pane, only the first row of squares (representing biosensors) is selected as tips will be regenerated between assays. In the bottom left pane, ensure all wells programmed in the first tab are highlighted blue indicating they will be used in the assay and that the assay was properly programmed in the second tab. In the right pane, ensure the proper tip type is selected. In the example assay, the protein is His-tagged so Ni-NTA tips are selected. If tip regeneration appears to be not working properly, see Notes 3-6.

5. In the fourth tab, use the arrows at the top right to step through and review the assay programming. If anything doesn't look correct, return to the appropriate tab and make corrections. Items to pay close attention to are the following: step time, step position, and step type.

6. In the fifth tab, use the top left pane to select a save destination for your data. In the middle pane, enter $25^{\circ} \mathrm{C}$ in the temperature box and ensure the plate delay is set to 600 seconds to permit the solutions plate to have sufficient time to equilibrate to the specified temperature. The temperature can be adjusted from $25^{\circ} \mathrm{C}$ per Note 7.

7. Do not press the GO button at this time. The plates and biosensors are programmed but still need to be physically prepared.

\subsection{Hydrate the Biosensors}

1. Using a spare biosensor box, remove the green tip rack and place a black 96 well plate into the bottom of the box. Fill Column 1 using $250 \mu \mathrm{L}$ of your POI buffer. In this example, it would be p53 buffer. In all remaining wells add $250 \mu \mathrm{L}$ ultrapure water to greatly reduce evaporation losses from your assay plate during the experiment. If you do not have a spare biosensor box, omit the addition of water from the extra wells.

2. Replace the green biosensor tray. Transfer a set of tips into the first column. Ensure the end of biosensor tip does not touch anything including the green tip tray. Any contact directly with the end of the tip will irreparably damage the 
biosensor. Discard any compromised tips in glass discard. After all tips have been transferred, either note the time or start a timer counting up. Additionally, return the unused biosensor back into their foil pouch with desiccant as soon as possible to protect them from moisture and light. The biosensors MUST hydrate in buffer for at least 10 minutes and be used within 24 hours of hydration.

Immediately place the hydrating biosensor box into the left side of the Octet and close the door to protect the biosensors from light. Do NOT leave the lid on the hydrating tips as this can break the optics box of the Octet.

\subsection{Filling the 96 Well Plate}

1. Dilute the protein stocks. Dilute GroEL to $0.5 \mu \mathrm{M}$ in GroEL buffer for a total of $2 \mathrm{~mL}$ and place tube on ice. Dilute your POI to an appropriate dilution and place on ice. In our example assay, p53 is diluted to $0.4 \mu \mathrm{M}$ which was selected as it produces a protein loading signal of $1 \mathrm{~nm}$ as well as being within the protein's linear response range, which is critical and discussed in Note 8.

2. Prepare dilutions of denaturant. Dilute the stock denaturant in protein buffer. In this example, the denaturant is urea. For urea, the dilutions range $0 \mathrm{M}$ to $7 \mathrm{M}$ by $1 \mathrm{M}$ steps. For discussion of stronger denaturants, see Note 9.

3. Fill the columns of the black 96 well assay plate with $250 \mu \mathrm{L}$ of the appropriate solution as planned in step 3.1.2. The order of addition should be solutions, denaturant, and finally proteins. Specifically, add POI buffer (this example p53 Buffer) to well Columns 1, 3, \& 11. Add GroEL Buffer to well Columns 5, 7, \& 9. Add $10 \mathrm{mM}$ glycine, $\mathrm{pH} 1.7$ to well Column 8 . Add $10 \mathrm{mM} \mathrm{NiCl}_{2}$ to column 10. Add ultra-pure water to unused column 12. Next, add the denaturant range with 0M urea in Row A through 7M urea in Row H. Finally, add diluted POI in well Column 2 and diluted GroEL to well Column 6.

4. Transfer the filled assay plate to the right side of the Octet, orienting the A1 position at the back right. Immediately close the door after.

\subsection{Running the Experiment}

1. Once the machine has been programmed, the biosensors hydrated, and the assay plate filled, click the GO button in the fifth tab to initiate the experiment following the programmed delay. Example data output is shown in Figure 4.

\subsection{Data Analysis}

1. Open the Data Analysis program provided with the Octet RED96. In tab 1, select your data folder to load you .frd data files. In tab 2, select the radio button option for "All Steps Aligned". Click the "Export Aligned Steps (.csv files) to output the raw data in a file which can be read with Microsoft Excel. Open the exported data with Excel. To normalize the GroEL association against the p53 (or POI) loading, the endpoint of GroEL association is divided by p53 (or POI) binding, as long as it is within the linear range (see Note 8). 
2. Plot the denaturant concentration on the $x$-axis and normalized GroEL binding on the y-axis to yield the kinetically controlled denaturant isotherm. Comparison between 50\% GroEL binding and denaturant concentration will yield information about a compound's ability to stabilize or destabilize the POI, corresponding to a right-shift or left-shift respectively of the 50\% GroEL binding denaturant concentration. The compounds should be added to the baseline before denaturant and during the denaturant solutions. Before testing compounds, it is important to establish if stabilization is possible. To test this, a general osmolyte stabilizer (sucrose or glycerol) should be included during the denaturant step. Figure 5 demonstrates stabilization of two proteins using osmolytes (4M glycerol and $1 \mathrm{M}$ sucrose).

3. Modeling of the data can permit quantitative comparisons between conditions, as was presented in the previous point. Additionally, if the model is a sigmoidal curve, this indicates cooperative denaturation. Most proteins fold and unfold in a cooperative manner. If the protein lacks native structure, this cooperativity will be not present. For alpha-synclein, which is generally thought to be unfolded and lacking structure in solution, the sigmoidal response to guanidine denaturation in Figure 6 indicates cooperative unfolding, suggesting at least partially folded structure in the native state.

\section{Notes}

1. Denaturant Pulse Length. A denaturant pulse length of 5 minutes ( 300 seconds) is applicable for most proteins. If the normalized GroEL binding response is at maximum at low denaturant, a shorter denaturant time (as short as 1 minute) will right-shift the isotherm such that the transition is observed at lower denaturant concentrations. Additionally, if the protein does not appear to have any GroEL binding even at maximal denaturant concentration, the denaturant pulse length can be increased to left-shift the isotherm enabling observation of the protein unfolding. We have tested pulses up to 10 minutes and found these to yield useful responses.

2. Wash Step Length. If refolding of the protein during the short (10s) wash prior to GroEL association, the length can be shortened. Wash steps from 0 s to 60 s have been examined in our lab. We found very little differences between 10s and 60s for anti-GST antibodies [1]. In addition, the Chaudhuri group has examined refolding on the tip using GroEL as the readout concluding the refolding rate is slow with only $40 \%$ refolding after $15 \mathrm{~m}$ at $25^{\circ} \mathrm{C}$ [2]. Therefore, it is not recommended to adjust this step as it is very unlikely your POI is refolding during the short wash step.

3. Protein Purity. The first item to check if inconsistent loading or GroEL binding using regeneration is protein purity. Impure protein samples have been observed by our lab to produce large variation between replicates.

4. Protein Stability. If protein purity has been checked and is not the confounding factor, the second item to check is the aggregation state of the POI. Because the 
triplicate assay requires over an hour of time at $25^{\circ} \mathrm{C}$, aggregation can occur particularly for proteins with marginal stability at this temperature. In order to prevent aggregation, include either $1 \mathrm{M}$ sucrose or $1 \mathrm{M}$ glycerol into the loading solution. This may prevent/slow down aggregation during the triplicate assay; however, the inclusion of either osmolyte will kinetically slow the loading rate, which requires a higher concentration of protein to achieve the same signal. In some special cases, an endogenous stabilizer can be used to stabilize the protein in solution without affecting the kinetics of loading (e.g. GTP stabilizes the nucleotide binding domain of CFTR protein [1] or p53 stabilization by CDB3, a peptide derived from the endogenous p53 binding protein 2 [3]).

5. Alternative Biosensor Regeneration Methods. If acid induced aggregation is suspected, regeneration using imidazole as a His-tag competitor can be attempted. In place of the acid/neutral \& and nickel steps, program a single 2minute incubation in $300 \mathrm{mM}$ imidazole. Alternatively, inclusion of a detergent (up to $0.05 \%$ Tween-20) in the neutralization buffer during regeneration can assist in regeneration.

6. Using Multiple Tips Instead of Biosensor Regeneration. If the loading response is not consistent after regeneration, one likely cause is incomplete regeneration or acid induced aggregation, which we have observed in some cases. To avoid regeneration complications, multiple tips can be used for obtaining triplicate runs to quantitatively evaluate the variation between runs. To use multiple tips, three modifications need to be made. First, when programming the assay in Step 3.1.3, select the radio button for "Add as a new assay" when replicating the assay. This forces the machine to use a new set of tips each assay run. Second, when hydrating biosensors, fill the first three columns with protein buffer in the 96 well plate and with biosensors. Third, the regeneration steps can be omitted from the assay steps.

7. Temperature of the Denaturant Pulse Assay. The temperature of the denaturant pulse assay may be raised from $25^{\circ} \mathrm{C}$ to a more physiological temperature of $37^{\circ} \mathrm{C}$. However, the protein linear response range, the concentration for $1 \mathrm{~nm}$ loading, and the aggregation propensity will need to be reassessed at this higher temperature as any kinetic rate will be increased with the increase in temperature. Additionally, the higher temperature will promote a greater amount of evaporation from the assay plate. For extended experiments this will change the functional concentrations. At $25^{\circ} \mathrm{C}$, only $5 \mu \mathrm{L}$ evaporation has been observed for the Octet RED96. The newer model Octet RED96e has greatly reduced evaporation at all temperatures and would be more appropriate to use for higher temperature experiments.

8. Linear Response Range. By loading the protein onto the tip within the linear response range, the GroEL binding response can be normalized to the POI loading. This accounts for minor variation in loading. However, outside this range normalization is not valid. For most proteins there are two linear ranges: one at very low concentrations and a second at higher concentrations, see Figure 
7. It is preferable it use the lower concentration linear range to limit any crowding or protein-protein interactions on the biosensor.

9. Other Denaturants. For proteins which are particularly stable as indicated by low GroEL binding at high denaturant concentrations, stronger denaturants can be used during the assay. In the same manner, a weaker denaturant can be used if the protein is denatured too easily. The denaturants we have tested are the following: urea < guanidine hydrochloride < guanidine thiocyanate. All the denaturants should be made fresh due to decomposition and/or evaporation, both of which will change the effective concentration.

\section{Acknowledgments}

This work was supported by NIH RO1AI090085 (M.T.F.), University of Kansas Innovation Center Grant (M.T.F.), 5R01GM112736 (J.K.), NSF EPScoR Kansas Board of Regents grant (M.T.F. and J.K.), NIH R21NS079881 (Q.Q.H.), the KBOR grant, T32GM008359 (P.T.O.), and Madison \& Lila Self Graduate Fellowship (A.J.M).

\section{References}

1. Lea WA, et al. Chaperonin-Based Biolayer Interferometry To Assess the Kinetic Stability of Metastable, Aggregation-Prone Proteins. Biochemistry. 2016; 55(35):4885-908. [PubMed: 27505032]

2. Pastor A, et al. Protein folding on biosensor tips: folding of maltodextrin glucosidase monitored by its interactions with GroEL. FEBS J. 2016; 283(16):3103-14. [PubMed: 27367928]

3. Friedler A, et al. A peptide that binds and stabilizes p53 core domain: chaperone strategy for rescue of oncogenic mutants. Proc Natl Acad Sci U S A. 2002; 99(2):937-42. [PubMed: 11782540] 


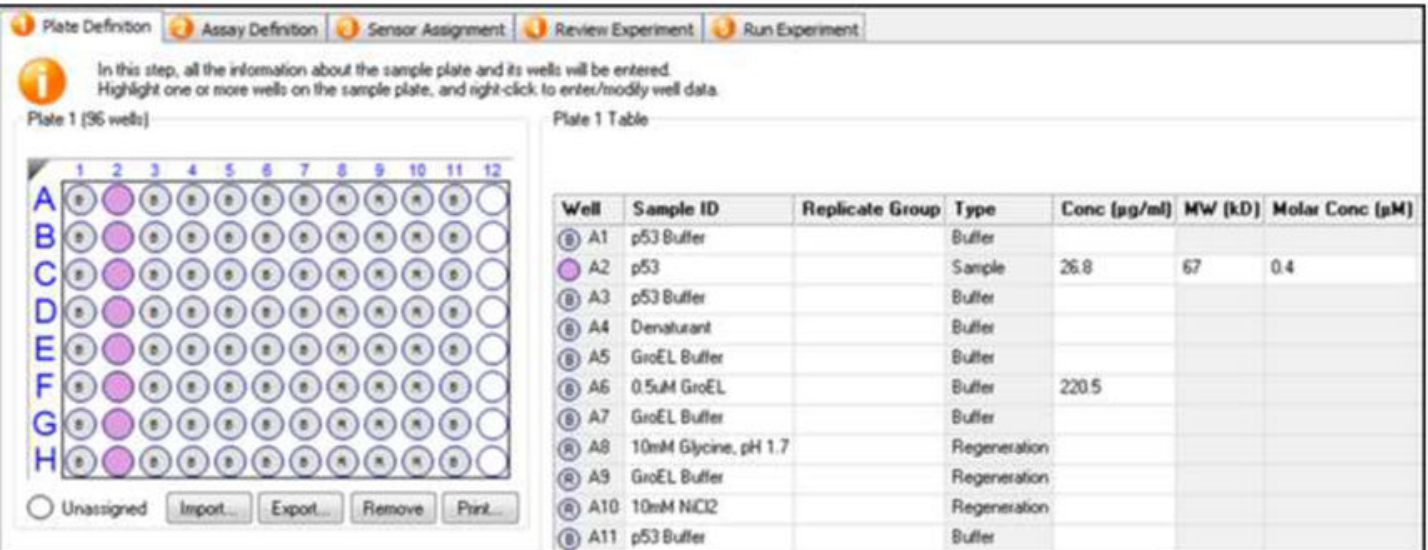

Figure 1. Tab 1 - Plate Definition

Screenshot displaying the setup for the standard denaturant pulse assay plate definition with plate on the left and descriptors on the right. 


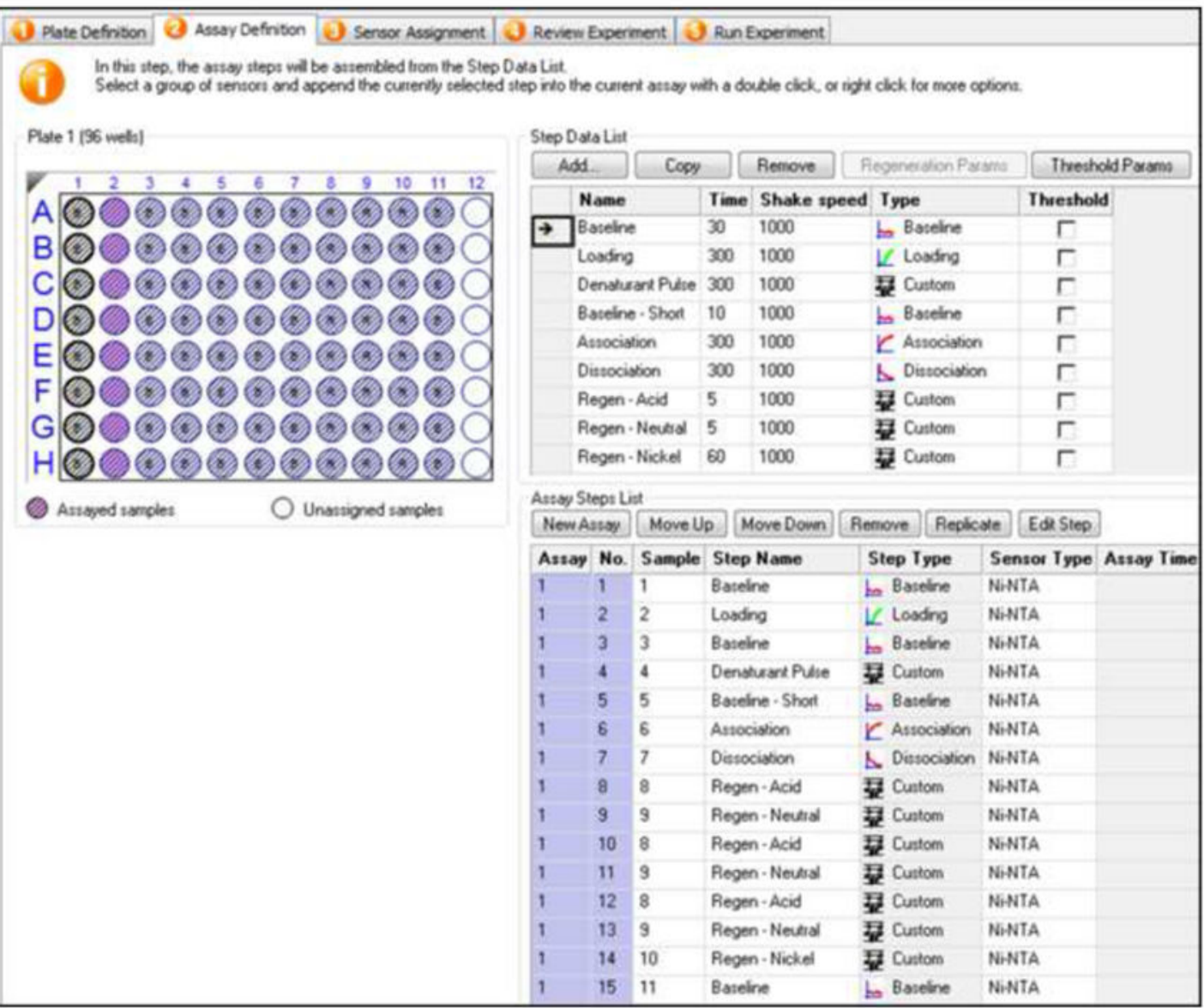

Figure 2. Tab 2 - Assay Definition

Screenshot displaying the setup for programming the denaturant pulse protocol with needed steps at the top right and the assigned steps at the bottom right. To program a step click on the step at the top right and double click on the well column to assign that step to the well. Only the first 15 steps are shown, which constitutes one full assay. 


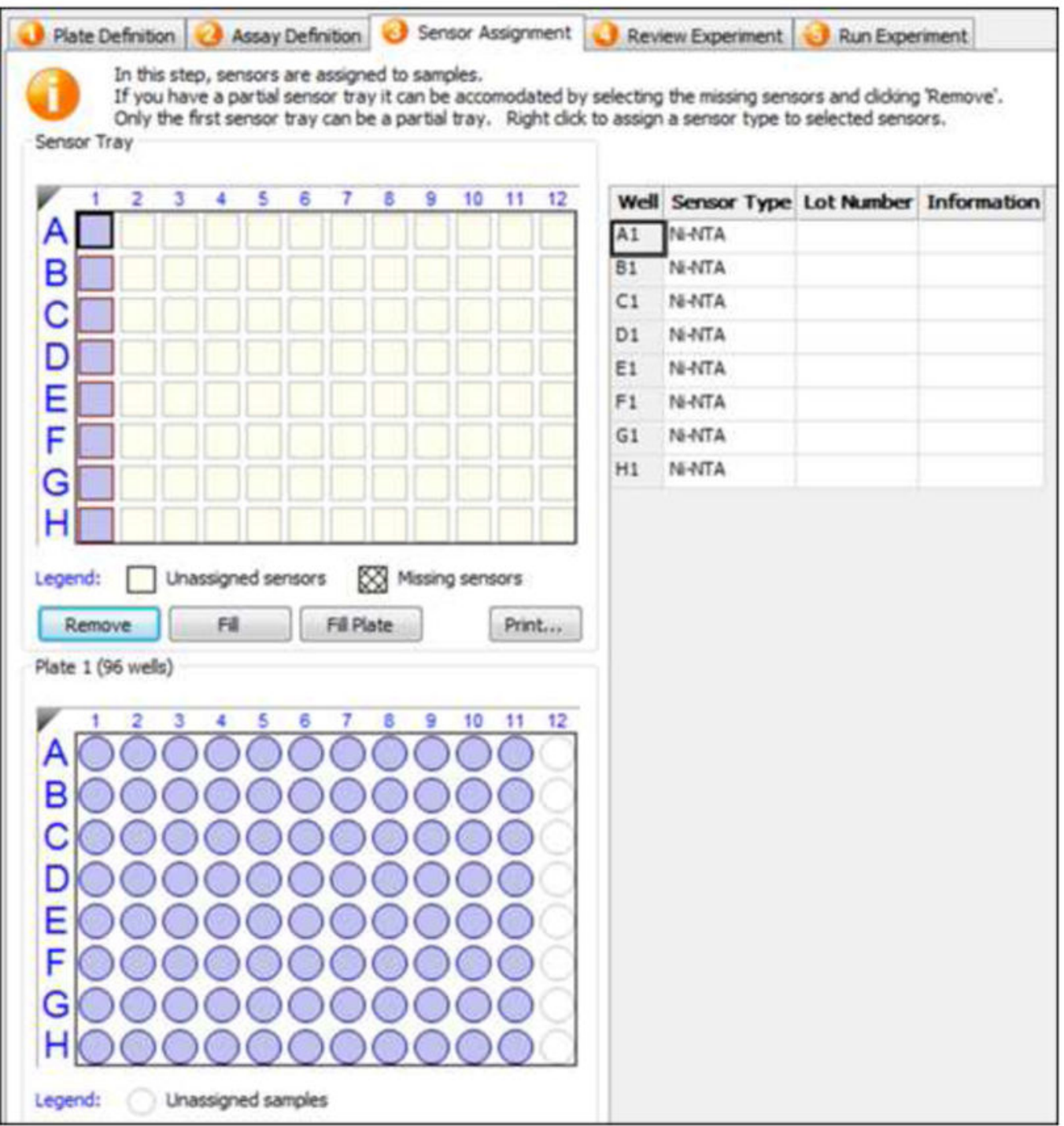

Figure 3. Tab 3 - Sensor Assignment

Screenshot displaying the sensor assignment tab. Biosensors are represented as squares in the top right pane. The plate assigned in tab 1 is in the bottom left pane. Sensor type is selected at the top right pane. 


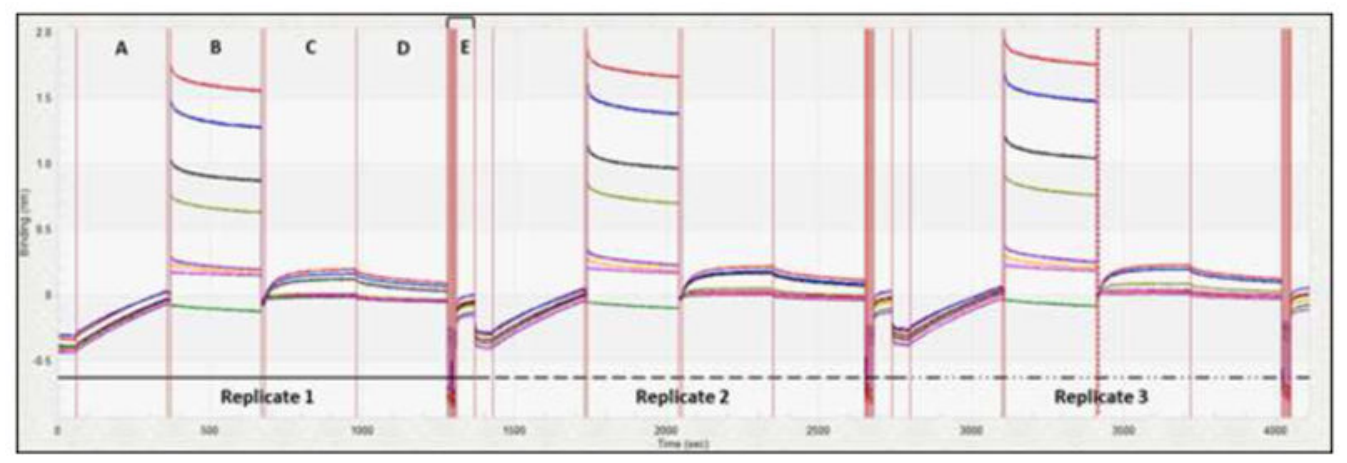

Figure 4. Sample Data

Sensogram trace of three assay replicates using acid/neutral regeneration. Key steps are labeled: A - POI loading, B - Denaturant Pulse, C - GroEL Binding, D - Dissociation, E Regeneration Steps. Each replicate assay is identified by bars at the bottom of the sensorgram. 

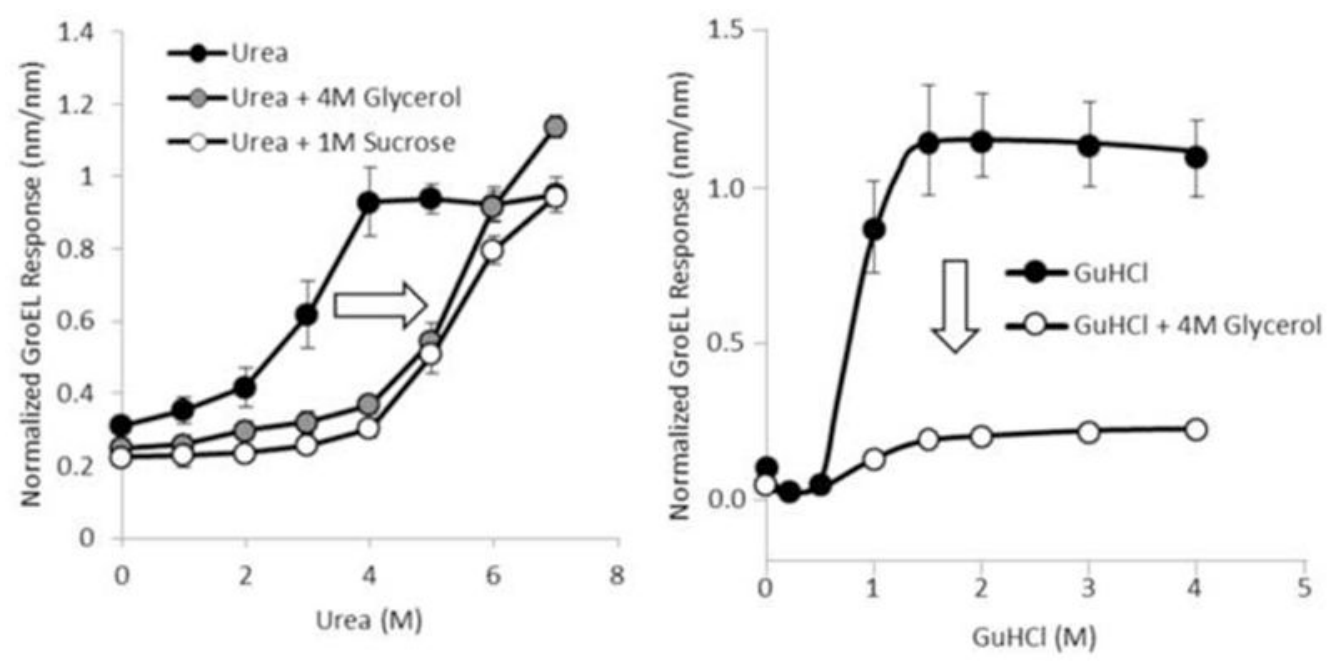

Figure 5. Denaturant Pulse Stabilization

Denaturant pulse isotherms demonstrating how inclusion of $1 \mathrm{M}$ sucrose or $4 \mathrm{M}$ glycerol in the urea (denaturant) solution can stabilize the POI. This stabilization is seen as a right-shift in the curve as indicated by the arrow for MalZ (left). Additionally, the GroEL binding may decrease upon stabilization as is seen for p53 using glycerol (right). Error bars are present on all data points of standard deviation for sample $n=3$. Adapted with permission from [1].

Copyright 2016 American Chemical Society. 


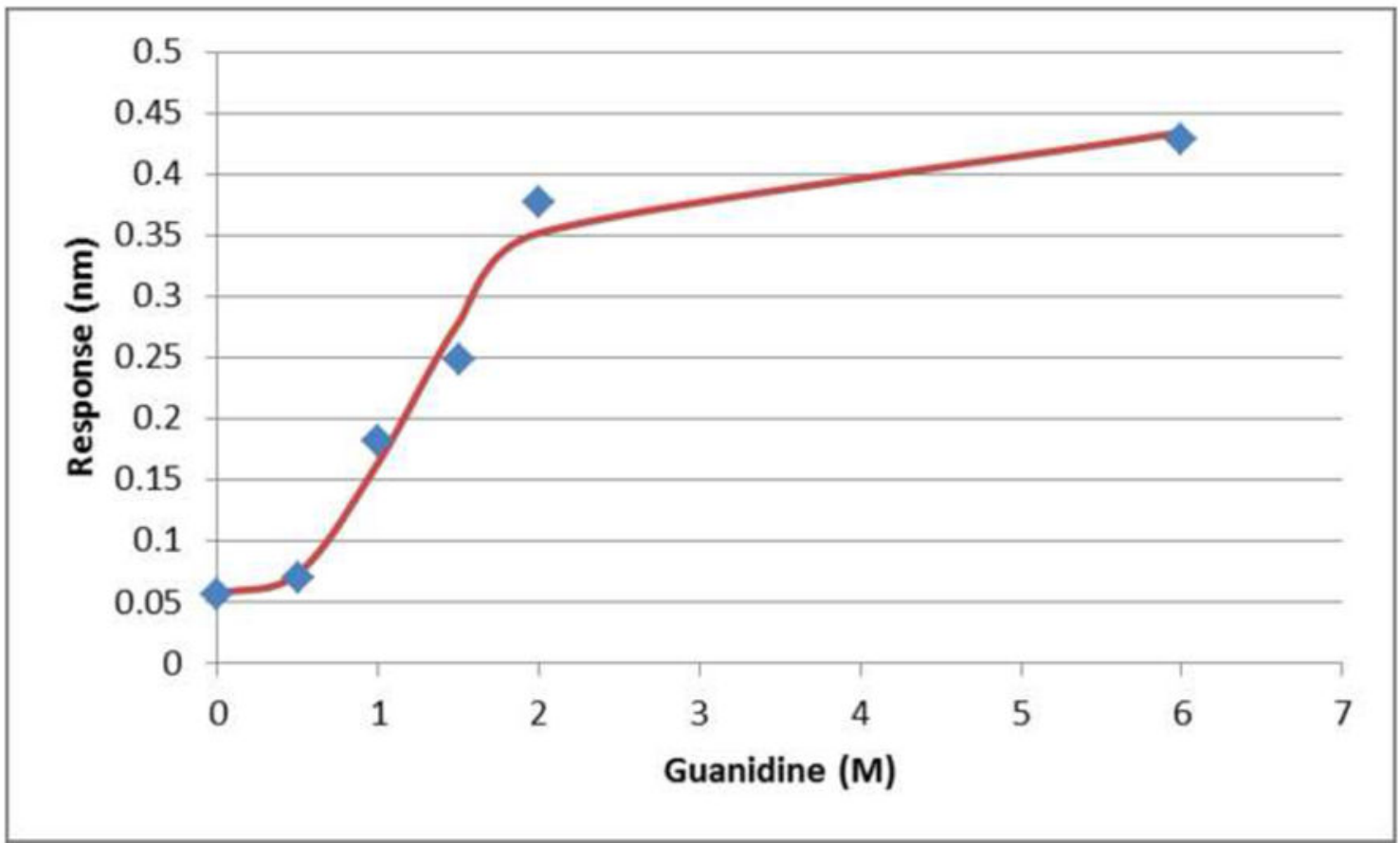

Figure 6. Sigmoidal Model of Denaturant Pulse

Plot of alpha-synuclein guanidine hydrochloride denaturant pulse with sigmoidal curve fit. Sigmoidal shape indicates a cooperative unfolding. 


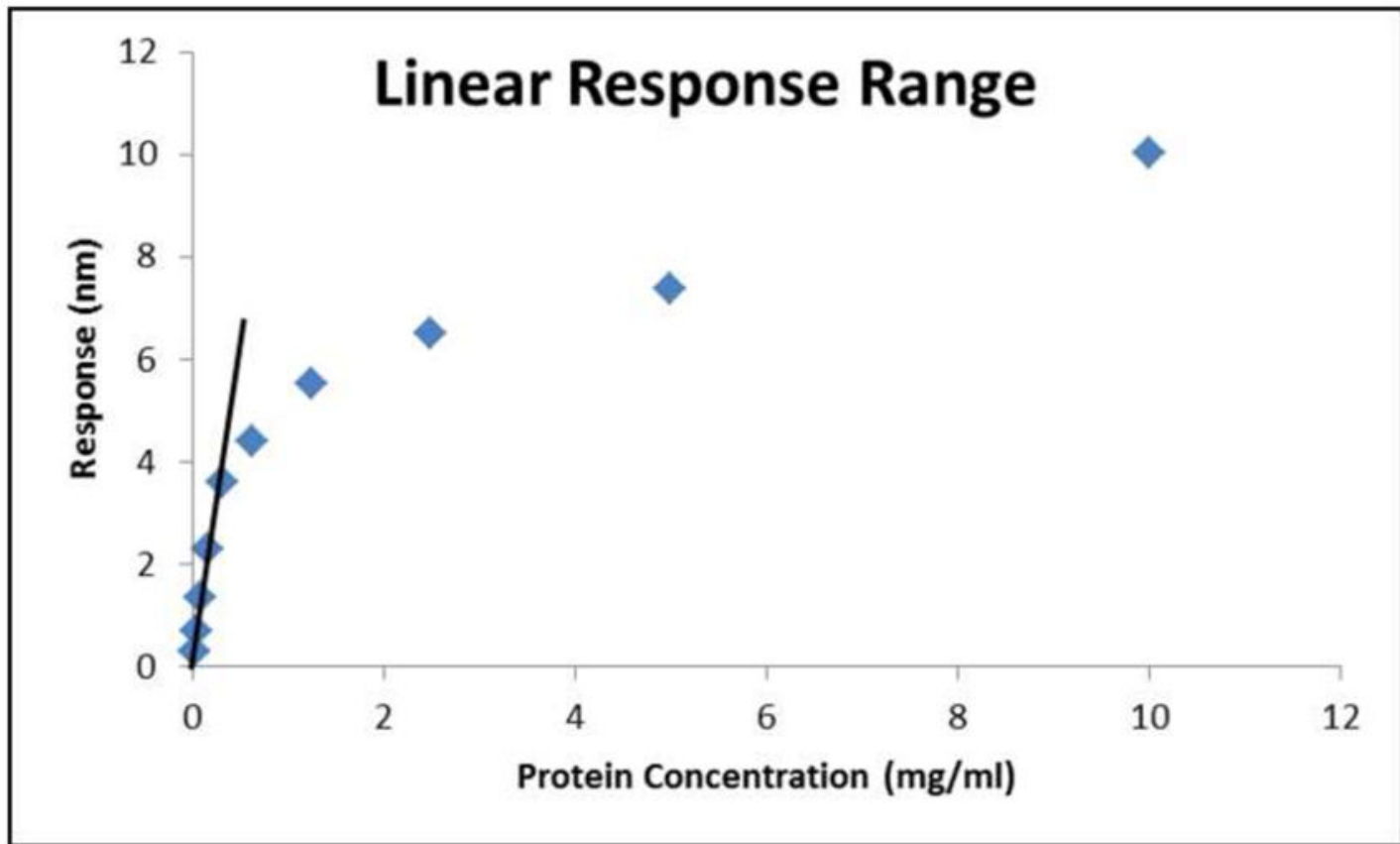

Figure 7. Linear Response Range Plot

Plot of Response versus protein concentration. For tetanus neurotoxin, the linear range can be seen at both very low concentration (solid line; $<0.5 \mathrm{mg} / \mathrm{ml}$ ) and at high concentrations $(>2 \mathrm{mg} / \mathrm{ml})$. These values are specific to every protein. 